\title{
Protection of HF Transmitters from Reflection Failure by Help of Semiconductor Isolators
}

\author{
L. Laurinavičius \\ Department of Electrical Engineering, Vilnius Gediminas Technical University \\ Sauletekio av.11, LT - 10223 Vilnius, Lithuania: e-mail: laurinavicius@el.vtu.lt; lagnel4@gmail.com
}

The fundamental problem of the transmitters protection against microwave energy reflections from aircraft antenna is discussed. The paper presents some applications of active and passive isolators and circulators as a tool for reflections protection. It has been stated that electrical properties of ferrite isolators and circulators grow worse at lower operational frequencies (below $50 \mathrm{MHz}$ ) and temperatures (cryogenic). Semiconductor magnetoplasma exhibits gyrotropic effects similar to those of magnetized ferrites and can solve the problem non-reciprocity in the $\mathrm{HF}$ range from 3 to $30 \mathrm{MHz}$, which is important for $\mathrm{HF}$ communication. The semiconductor device realization, optimal parameters, and operational temperature range, based on minimum forward loss $\alpha$ and maximum reverse attenuation $\beta$, were chosen. Major electromagnetic parameters of semiconductor isolators were determined.

Keywords: Protection, helicon, high frequency, magnetic field, non-reciprocity, semiconductor isolator, temperature stability

\section{INTRODUCTION}

Ferrite circulators and isolators are the key elements in modern microwave engineering. Their fundamental property of non-reciprocity is capable of simplifying the construction and improving the stability, efficiency and accuracy of radar, communication and testing systems.

The devices contain a core of ferrite biased by a static magnetic field. This field orients the electron spins within the ferrite to produce a gyromagnetic effect. The non-reciprocity occurs when radio frequency (RF) signal interacts with the precessing electrons to set up a standing-wave pattern within the core.
An isolator (Fig.1, $a$ ) is a passive non-reciprocal 2-port device which permits signal energy to pass through it in forward direction whilst absorbing energy in the reverse direction.

A circulator (Fig. 1, b) is a passive non-reciprocal device with 3 or more ports. Energy introduced into the first port is transferred to the second port, the others ports being isolated.

The frequency range within the non-reciprocal ferrite devices meets the guarantied specifications that are not less than $50 \mathrm{MHz}$.

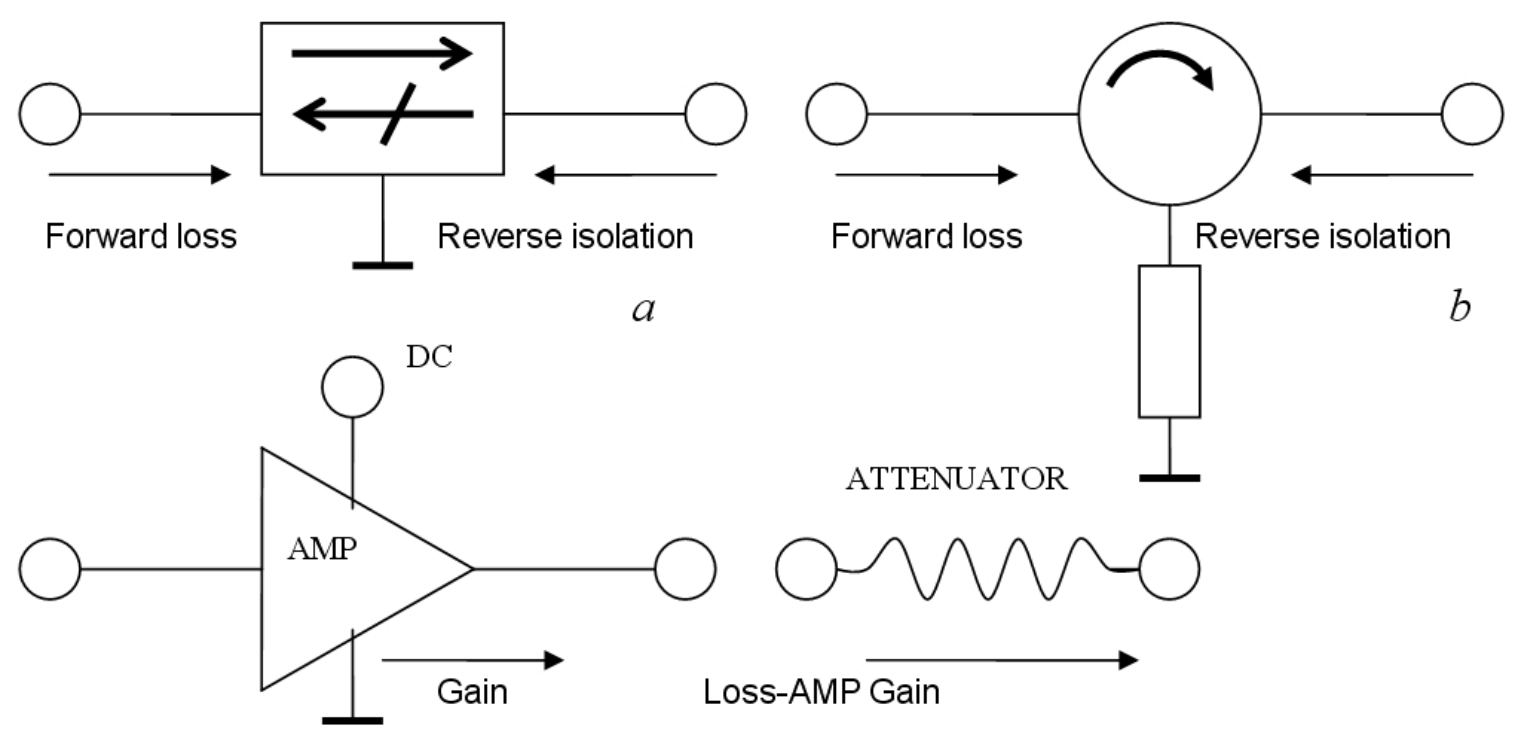

Hight reverse isolation

Fig.1 Passive isolator $(a)$, circulator $(b)$ and active isolator, using amplifier and attenuator $(c)$. 
Non-reciprocal devices are placed between the transmitter and antenna. RF signal can reach the antenna but the returning signal goes to the load (circulator) or dissipates at the core (isolator).

Insertion loss $\alpha$ (expressed in $\mathrm{dB}$ ) is the attenuation that results from inserting the device. It is also defined as the ratio of signal power at the output of the inserted device to the signal power at the input of the inserted device.

Isolation $\beta$ is the ratio, expressed in $\mathrm{dB}$, of the input power to output power for signal in the reverse direction.

Maximum power and temperature range are the are also important parameters for the non-reciprocal devices.

Electrical properties of ferrite isolators and circulators grow worse at lower operational frequencies (below $50 \mathrm{MHz}$ ) and temperatures (cryogenic).

Semiconductor magnetoplasma exhibits gyrotropic effects similar to those of magnetized ferrites and can solve the problem non-reciprocity in the $\mathrm{HF}$ range from 3 to $30 \mathrm{MHz}$, which is important for HF communication [1].

A different approach to the basic nonreciprocal mechanism is used in solid-state plasma devices. This approach makes use of solid-state plasma waves, so-called helicon waves, which are nonreciprocal by their nature [2].

An active isolator $($ Fig. $1, c)$ can also solve the protection problem. The attenuator and amplifier (Fig.1, c) were connected. The forward gain becomes zero while reverse isolation will be more than $20 \mathrm{~dB}$ [3]. The active devices have noise, input and output, linearity problems, but they are a useful solution over wide bandwidths, where ferrite and semiconductor isolators are unavailable.

\section{PhysicAl BACKGROUND OF InVESTIGATION}

Physical background of operation of the semiconductor isolator is based on the effect of dimensional resonance of helicon waves in a plate placed in the static magnetic field (see a Fig.2).

The propagation of magnetoplasma wave $k_{ \pm}$may be detected from the characteristic equation [4]

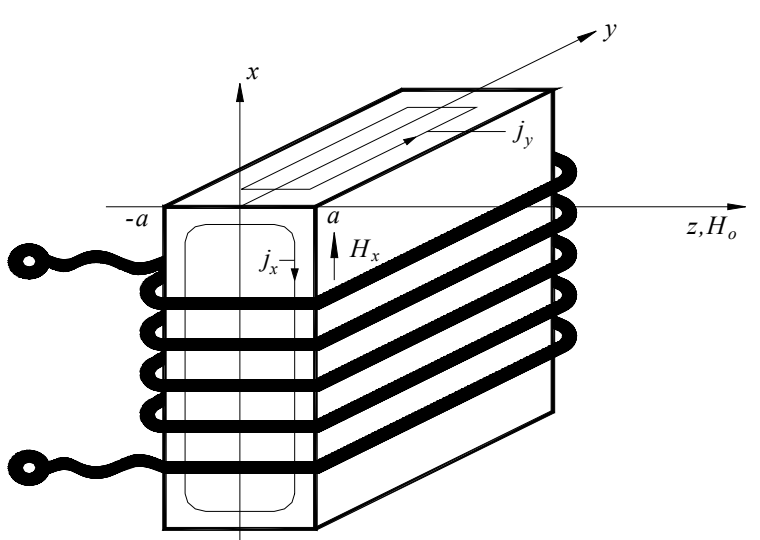

Fig.2 Conducting plate with an electric coil

$$
\begin{gathered}
c^{2} k_{ \pm}^{2}=\frac{\omega_{p}^{2} \omega}{ \pm \omega_{H}+i \tau^{-1}}, \omega_{p}^{2}=\frac{4 \pi N e^{2}}{m} \\
\omega_{H}=\frac{e H}{m c} .
\end{gathered}
$$

where $k$ is the wave vector, $\omega$ is frequency, $\omega_{H}$ is cyclotron frequency, $\omega_{p}$ is plasmas frequency, $H$ is magnetic field, $\tau$ is relaxation time and $m$ is effective mass carriers. Items with the argument $k_{+}$are caused by the helicon waves. In the case $\omega_{H} \tau$ $>>1$ we have

$$
k_{+}=\sqrt{\frac{\omega_{p}^{2} \cdot \omega}{\omega_{H} \cdot c^{2}}}\left(1-\frac{i}{\omega_{H} \tau}\right)
$$

And effective magnetic permeability

$$
\mu_{e f f}=\frac{\Phi_{x}}{2 a H}=\frac{t g_{-} a}{2 k_{-} a}+\frac{\operatorname{tg}_{+} a}{2 k_{+} a} .
$$

The $\mu_{\text {eff }}$ has a maximum in the case of resonance

$$
\left(\operatorname{Re} k_{+}\right) a=\frac{n \pi}{2}, n=1,3,5, \ldots
$$

\section{MOdEling OF THE SEMICONDUCTOR ISOLATORS}

We have developed a model of the helicon isolator. We considered the electromagnetic parameters of the helicon resonator material, dimensional effects, the impact of the mine carriers, and some other issues.

The main element of the represented model is the nonreciprocal transformer, characterized by the matrix of impedances.

The impedance matrix for the nonreciprocal transformer is calculated according to the formula [5].

$$
|Z|=\left|\begin{array}{cc}
z_{11} & z_{12} \\
z_{21} & z_{22}
\end{array}\right|=\left|\begin{array}{cc}
i \omega L_{1} & i \omega M_{21} \\
i \omega M_{12} & i \omega L_{2}
\end{array}\right|
$$

where $L_{1}=\mu_{\mathrm{L}} L_{01}$ and $\mathrm{L}_{2}=\mu_{\mathrm{L}} L_{02}$ are inductances of coils whose physical dimensions are approximately the same as helicon resonator size $M_{21}=-M_{12}=M_{\mathrm{L}} \mu_{\mathrm{T}}, M_{\mathrm{L}}^{2}=L_{01} L_{02}$. $\mu_{\mathrm{L}}$ and $\mu_{\mathrm{T}}$ are ,magnetic permeabilities" of the semiconductor core in parallel and perpendicular directions, when resonance $(n=1)$ appears

$$
\begin{gathered}
\mu_{\mathrm{L}}=1-4 / \pi^{2}\left(1+2 i \mathrm{Q} x_{m}\right) /\left[1+i \mathrm{Q}\left(x_{m}-x^{-1}\right)\right], \\
\mu_{\mathrm{T}}=4 u / \pi^{2}\left(1 /\left[1+i \mathrm{Q}\left(x_{m}-x_{m}^{-1}\right)\right],\right.
\end{gathered}
$$

where $u=R_{\mathrm{H}}|\mathrm{B}| / \rho=\operatorname{tg} \Theta, \Theta$ is the Hall angle and $R_{\mathrm{H}}$ is the Hall constant.

Quality $Q$ of the helicon resonator depends on the Hall constant $R_{\mathrm{H}}$ and magnetic induction $B$ 


$$
Q=1 / 2\left(1+u^{2}\right)^{1 / 2} \text {. }
$$

The angular frequency of the mine dimensional resonance (resonance Fabry-Perot) $\omega_{\mathrm{r}}(0)$

$$
\omega_{\mathrm{r}}(0)=\left(2 \rho Q / \mu_{0}\right)\left(\pi^{2} / d^{2}\right)
$$

If $u=\mu B>1, \omega_{r}(0)<<\omega_{H}$, where $\mu$ is carrier mobility, the frequency of the mine dimensional resonance can be written in simple form [5],

$$
f_{\mathrm{r}}=A B /\left(N d^{2}\right),
$$

where $A=7,82 \cdot 10^{24}$ is the constant, $N$ is density (concentration) of the carrier of the semiconductor material. The quadripole of this type will resemble a gyrator. The half wave helicon resonator with the inductance coils represents a passive device - the nonreciprocal transformer with nonreciprocal phase shift equal to $180^{\circ}$ in the direct $(+\pi / 2)$ and reverse $(-\pi / 2)$ directions.

The rate of coupling between the inductance coils and the resonator depends on $Q$ factor of the dimensional resonator, number of turns in the inductance coils, on the filling of coils by core, and the cross section between the coils [5].

By transmitting the helicon waves through a semiconductor plate the effect of a resonator shape and size on resonance characteristics was investigated. It was found that $d / a<0.2$, where $a$ is the least value of the plate width, resonance frequency may be determined by the equation (10), used for semi - infinite resonator.
In Fig. 3 we can see calculated by (7) the frequency responses of the real and imaginary parts of the magnetic permeability $\mu_{\mathrm{T}}$ of a semiconductor core - helicon resonator where $U$ is invariant of the isolator system and for ideal isolator $U=1$, because insertion loss $\alpha=0$.

For the nonreciprocal low pass filter (L1-C2-L2) isolator we have $U^{2}=1 /\left(1+1 / u^{2}\right)$.

The curves 1, 2, 3, 4 cover different electron densities $N$ of the semiconductor: $2,3 \cdot 10^{-22} \mathrm{~m}^{-3}(4) ; 2,0 \cdot 10^{-22} \mathrm{~m}^{-3}(3) ; 1,7 \cdot 10^{-22}$ $\mathrm{m}^{-3}(2) ; 1,4 \cdot 10^{-22} \mathrm{~m}^{-3}(1)$.

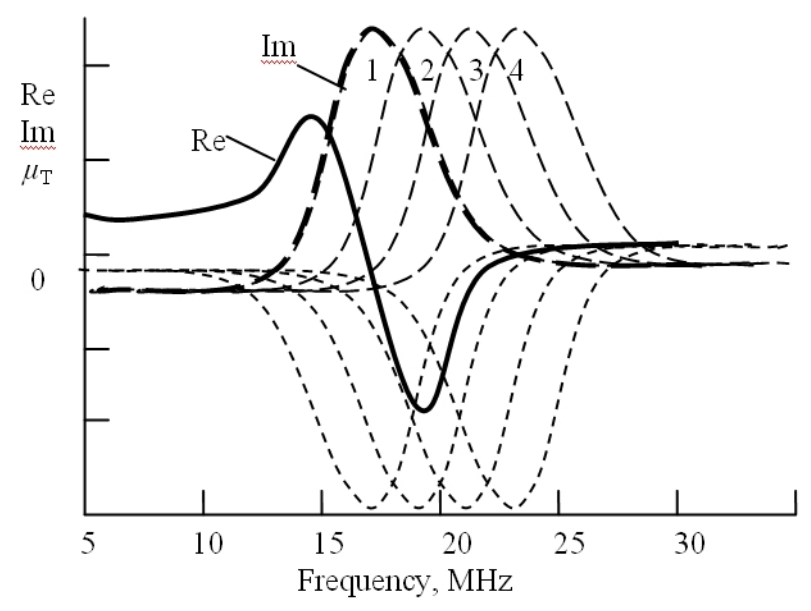

Fig.3 Calculated frequency responses of magnetic permeability $\mu_{\mathrm{T}}$
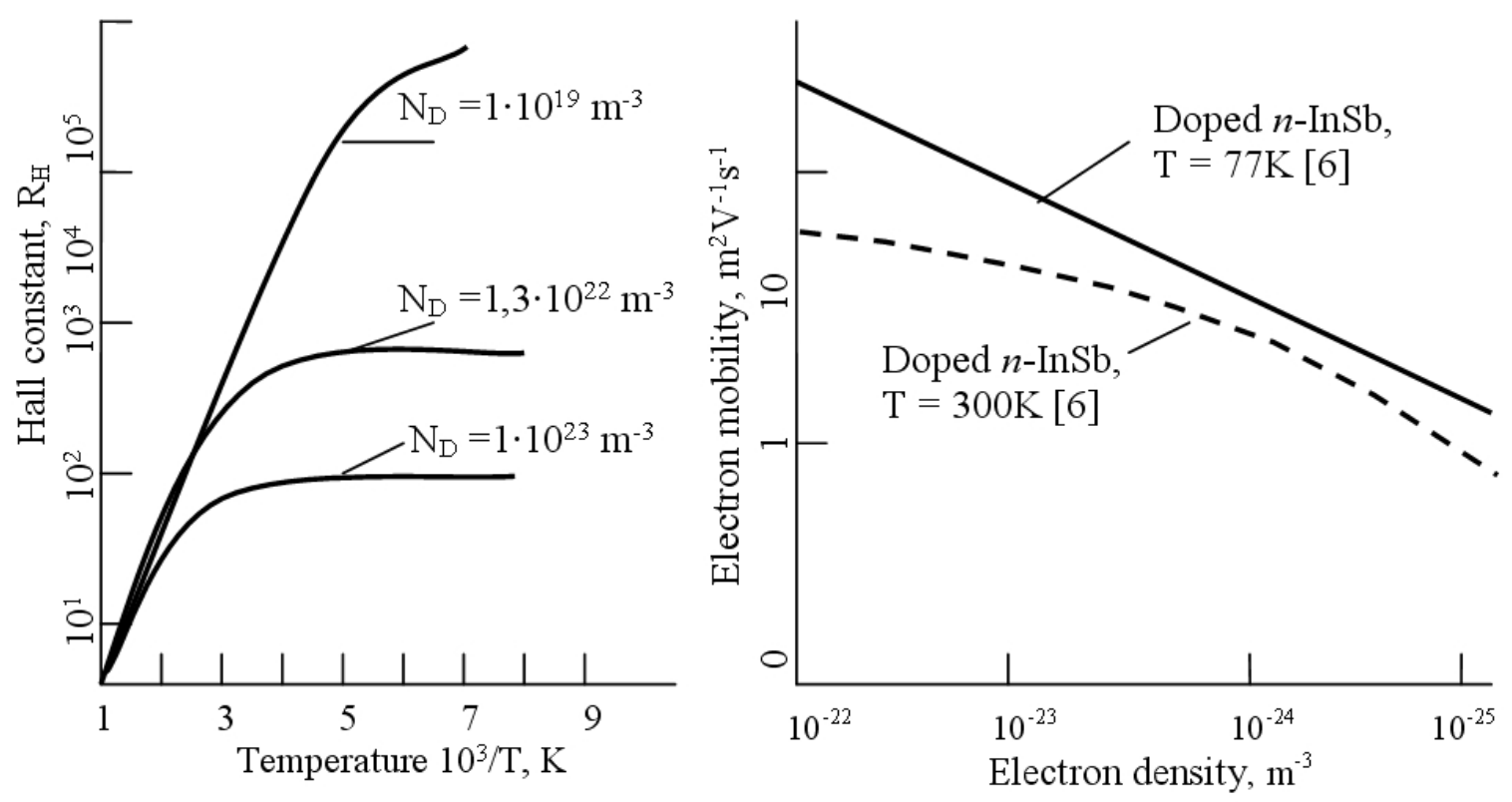

Fig.4 Dependencies of the Hall constant $\mathrm{R}_{\mathrm{H}}$ of $\mathrm{n}$-InSb, doped with tellurium, of temperature and electron mobility $\mu$ of density $N$ doped $n$-InSb. 
In Fig. 4 typical dependencies of the Hall constant $R_{\mathrm{H}}$ of $n$ InSb, doped with tellurium, of temperature are shown. We can see, that only for doped $n$-InSb (density donors $N_{\mathrm{D}}=1,0 \cdot 10^{23}$ $\mathrm{m}^{-3}$ ) in temperatures $T \sim 300 \mathrm{~K}$ the Hall constant $R_{\mathrm{H}}$ and density $N$ are stable. For doped n-InSb (density donors $\mathrm{N}_{\mathrm{D}}=1$, $3 \cdot 10^{22} \mathrm{~m}^{-3}$ ) stable range of $\mathrm{R}_{\mathrm{H}}$ does not exceed $250 \mathrm{~K}$.

If mine carrier (electron) density $\mathrm{N}>2 \cdot 10^{-23} \mathrm{~m}^{-3},(\mathrm{~T}=$ $300 \mathrm{~K})$, the temperature stability is better, but mobility of electrons (Fig.4) is less than $4 \mathrm{~m}^{2} \mathrm{~V}^{-1} \mathrm{~s}^{-1}$. High magnetic fields are necessary.

We can supplement the nonreciprocal phase transformer with new circuit elements depending on the schematic realization of the isolator as nonreciprocal LC-filters of different types. The isolators (Fig.5) can be made up with nonreciprocal low pass filter (L1-C2-L2), nonreciprocal high pass filter (L1-C4-L2) or with band pass or band rejection filters $[7,8]$.

The scattering matrix for the isolator:

$$
|\mathrm{S}|=\left|\begin{array}{cc}
\mathrm{S}_{11} & \mathrm{~S}_{12} \\
\mathrm{~S}_{21} & \mathrm{~S}_{22}
\end{array}\right|=\left|\begin{array}{cc}
0 & U \mathrm{e}^{i \xi} \\
0 & 0
\end{array}\right|,
$$

where $U$ is invariant of the isolator system and for ideal isolator $U=1$, because insertion loss $\alpha=0$.

The electrical parameters of the circuit components (for example the value of capacitance $\mathrm{C} 2$ ) can be found by minimum loss $\alpha$.

We can calculate direct loss $\alpha$ and reverse attenuation $\beta$ in decibel by the formula. where $P_{\mathrm{G}}, Z_{\mathrm{G}}$ are power and impedance of the generator, $P_{\mathrm{L}}$ is output power and $Z_{\mathrm{L}}$ is load impedance and $Z_{\mathrm{IN}}=\mathrm{V}_{\mathrm{IN}} / \mathrm{I}_{\mathrm{IN}}$; $Z_{\mathrm{T}}=\mathrm{V}_{\text {IN }} / \mathrm{I}_{\text {OUT }}$.

\section{Problem of Temperature Stability. Choice and OPTIMIZATION OF THE MATERIAL}

According to (10) the density of carrier should be temperature stable. The most appropriate semiconductor is the $n$-type indium antimony ( $n-\mathrm{InSb}$ ) doped with tellurium. If the electron density is higher, the temperature stability is better. However, an increase in concentration of donor impurity results in diminishing the electron mobility and the resonator quality factor. All this will cause the isolator forward loss to increase. The temperature characteristics of helicon resonators were considered for a low-band filter isolator because loss of these isolators is lower.

Based on the chosen model, we have calculated the main characteristics of the isolator: direct loss $\alpha$ and reverse attenuation $\beta$ in the wide range of temperatures and frequencies. It has been shown that for the development of mobile non-reciprocal devices a semiconductor material of high carrier mobility $\mu>4 \mathrm{~m}^{2} \mathrm{~V}^{-1} \mathrm{~s}^{-1}$ and high mine carrier density $N>10^{-22} \mathrm{~m}^{-3}$, $(\mathrm{T}=300 \mathrm{~K})$ is needed. The electrical parameters of semiconductors should be stable in operational temperature range. Te - doped $n$-InSb, semiconductor alloy $\mathrm{Cd}_{\mathrm{X}} \mathrm{Hg}_{1-\mathrm{X}} \mathrm{Te}(\mathrm{x} \sim 0,1)$ and anisotropic alloy $\mathrm{Bi}_{1-\mathrm{X}} \mathrm{Sb}_{\mathrm{X}}(\mathrm{x} \sim 0,1)$ offers valuable properties.

In our calculations, we considered the frequency responses and temperature characteristics of the helicon resonator material, the helicon resonator geometry, physical parameters and dimensions of the inductance coils, characteristics of insulation between the coils, parameters of the transformer filling, electrical parameters of the circuit components, etc.

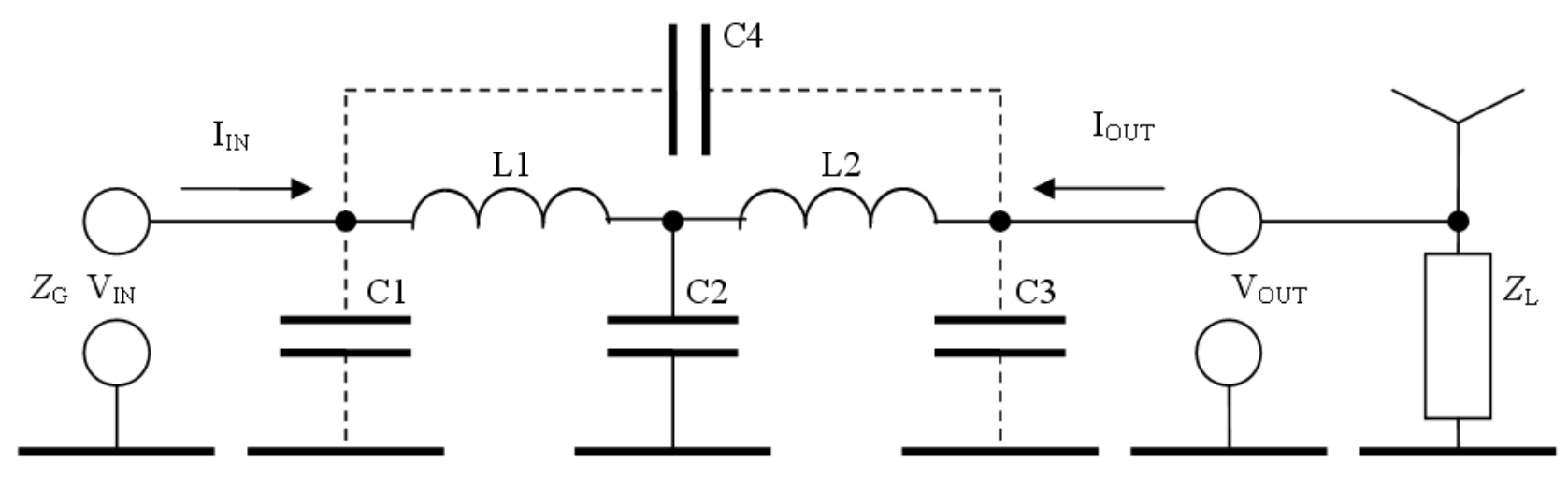

Fig. 5. Semiconductor isolator 


\section{CONCLUSIONS}

Nonreciprocal passive devices as isolators and circulators based on ferrite medium are used in microwave engineering and wireless communication.

One of the problems is protection of the transmitters against microwave energy reflections from aircraft antenna in decametric range of waves. The low-frequency limit of applicability of ferrite nonreciprocal devices is $50 \mathrm{MHz}$. At lower frequency range, application of nonreciprocal ferrite devices is questionable. The difficulties arise from the absence of ferrite materials with the necessary properties. In the metric and decametric range of waves, the helicon devices may serve as a substitute for ferrite nonreciprocal devices. The main point is that the helicon devices use the gyroelectric effects in semiconductors instead of the gyromagnetic one in ferrites.

A mathematical model describing the electrical properties of non-reciprocal quadrupole has been presented. It has been shown that for the development of mobile non-reciprocal devices a semiconductor material of high carrier mobility $\mu>$ $4 \mathrm{~m}^{2} \mathrm{~V}^{-1} \mathrm{~s}^{-1}$ and high mine carrier density $\mathrm{N}>10^{-22} \mathrm{~m}^{-3},(\mathrm{~T}=$ $300 \mathrm{~K})$ is needed.

The electrical parameters of semiconductors should be stable in operational temperature range.

The characteristics of the helicon isolators containing a nonreciprocal transformer with helicon dimensional resonator are calculated. The experimental results are in compliance with calculations that confirm adequacy of the used model.

\section{REFERENCES}

[1] NATO Research \& Technology Organisation (2007). RTO-TR-IST-050 - HF Interference, Procedures and Tools.

[2] Gremillet, J. (1964). Propagation des ondes metriques et decametriques dans les semi-conducteurs et presence d'une induction magnetique continue. Effect Helicon. Ann. de Radioelect. no. 76, 122-150.

[3] Polivka, I. (2006). Wideband UHF/microwave active isolators. High Frequency Electronics, 5 (12), 70-71 (design notes).

[4] Jankauskas, Z., Laurinavičius, L. (2002). Magnetic and electric excitation of magnetoplasmic waves. Electronics and Electrical Engineering, no. 2 (37), 32-34.

[5] Laurinavičius, L. (1995). Non-Reciprocal and Control Devices Based on the Semiconductor Magnetoplasma. Vilnius: Technika. (in Russian)

[6] Litwin-Staszewska, E., Szymanska, W., Piotrzkowski, P. (1981). The electron mobility and thermoelectric power in InSb at atmospheric and hydrostatic pressures. Physica Status Solidi B, 106 (2), 551-559.

[7] Vountesmery, V.S., Vountesmery, Y.V. (2007). Temperature characteristics of broadband helicon isolators for meter and decameter waves. IEEE Transactions on Microwave Theory and Techniques, 55 (10), $2097-2102$.

[8] Laurinavičius, L. (1982). High frequency transformer. U.S.S.R. Patent 930398 BI 19. 